\title{
From Scintillator-based Detector to Direct Electron Detector: High Performance of Next Generation of Camera for In-situ TEM Testing and TEM Imaging
}

\author{
Hua Guo ${ }^{1}$, Liang Jin ${ }^{1}$, Penghan $\mathrm{Lu}^{2}$, Zhangjie Wang ${ }^{2}$,Zhiwei Shan ${ }^{2}$, Benjamin Bammes ${ }^{1}$, Michael \\ Spilman $^{1}$, and Robert Bilhorn ${ }^{1}$ \\ 1. Direct Electron, LP, San Diego, CA, USA. \\ 2. Center for Advancing Materials Performance from the Nanoscale (CAMP-Nano), Hysitron Applied \\ Research Center in China (HARCC), XJTU-Hitachi High-Tech Research \& Development Center \\ (XHRDC), State Key Laboratory for Mechanical Behavior of Materials, Xi'an Jiaotong University, \\ $\mathrm{Xi}$ 'an 710049 , China
}

Transmission electron microscopy (TEM) is a powerful technique for characterizing materials at super high spatial resolution. In the past decades, many TEM related techniques like EDX, EELS, STEM, Cscorrection and HRTEM have been well developed [1]. As a result, both chemical and structural information has been obtained at atomic resolution for many important specimen types, which makes transmission electron microscopes indispensable scientific instruments in the modern laboratory. Recently, in-situ TEM testing techniques have attracted intense research attention, especially in the materials science community. For instance, the propagation of defects has been observed directly in compression tests of $\mathrm{Ni}$ nanopillars [2]; atomic rearrangement has been recorded when straining $\mathrm{Au}$ nanostructures [3]; a new deformation mode has been found in small amorphous alloy samples in tensile tests [4]; and even crystal growth processes and chemical reactions have been captured in liquid environments [5]. Such in-situ testing experiments provide important direct evidence about materials deformation mechanisms and chemical reactions, but also introduce new instrumentation challenges in the TEM. These include undesired dynamic specimen processes (e.g., drift, beam-induced motion, charging, radiation damage, etc.), insufficient field of view, insufficient frame rates, and inefficient electron detectors [6].

With the goal of overcoming many of these obstacles for the life sciences, Direct Electron (San Diego, CA, USA) introduced the first large-format Direct Detection Device (DDD ${ }^{\circledR}$ ) in 2008, as the culmination of academic and industrial partnerships working through six generations of sensor development beginning in 2001 [7]. Recently, Direct Electron released its new 20-megapixel "DE-20" camera system, representing the ninth generation of DDD development and providing the largest field-of-view of any available TEM direct detection camera [8]. Direct Electron has now released new tools for in-situ TEM that enable high-speed recording of large format, very high quality "movies" of dynamic processes.

Here, we show results from the DE-12 DDD camera with the new in-situ tools clearly observing the twining process in a $200 \mathrm{~nm}$ diameter $\mathrm{Cu}$ nanopillar in a quantitative compression test. Figure 1 shows five snapshots from a video stream recorded with the camera at a frame rate of 57 frames per second. Fig. 1 (a) is a TEM bright field image of the pristine $\sim 500 \mathrm{~nm}$ high $\mathrm{Cu}$ single crystal nanopillar. The primary twin, going through the whole specimen, started emerging in Fig. 1(b) at 8.25 sec, and formed completely in the subsequent $0.14 \mathrm{sec}$ as shown in in Fig. 1(c). Afterwards, the twin grew gradually until it ruptured, which took about $0.39 \mathrm{sec}$ as shown between Figs. 1(d,e). A FIB fabrication-induced damaged layer could be distinguished easily with a thickness of about $7 \mathrm{~nm}$ from the robust specimen. The layer was found to be flexible, peeling off at the surface where the primary twining happened as shown in Fig. 2, which is an enlarged view of the framed area in Fig. 1(e). Mechanical adhesion between 
the damaged layer and the nanopillar appears not to be strong at all. In this regard, it is reasonable to ignore the influence of the damaged layer for this quantitative mechanical test.

The very good signal-to-noise ratio (SNR) and wide dynamic range of individual frames taken from the movie, as illustrated by the observation of the FIB damage layer, illustrate the impressive capabilities of this new in-situ tool.

\section{References}

[1] D.B. Williams, and C.B. Carter, "Transmission Electron Microscopy: A Textbook for Materials", 2nd Ed. (Plenum Press, New York).

[2] Z.W. Shan et al, Nature Materials, 7 (2007) 115.

[3] H. Zheng et al, Nature Communications 1 (2010) 144.

[4] H. Guo et al, Nature Materials, 6 (2007) 735.

[5] H.G. Liao et al, Science, 345 (2014) 916.

[6] R.M. Glaeser, and R.J. Hall, Biophys J 100 (2011) 2331.

[7] L. Jin and R. Bilhorn, Microsc Microanal 16 (2010) 854-855.

[8] B.E. Bammes et al, Microscopy \& Microanalsis Conference Proceedings (2012) LB-36.
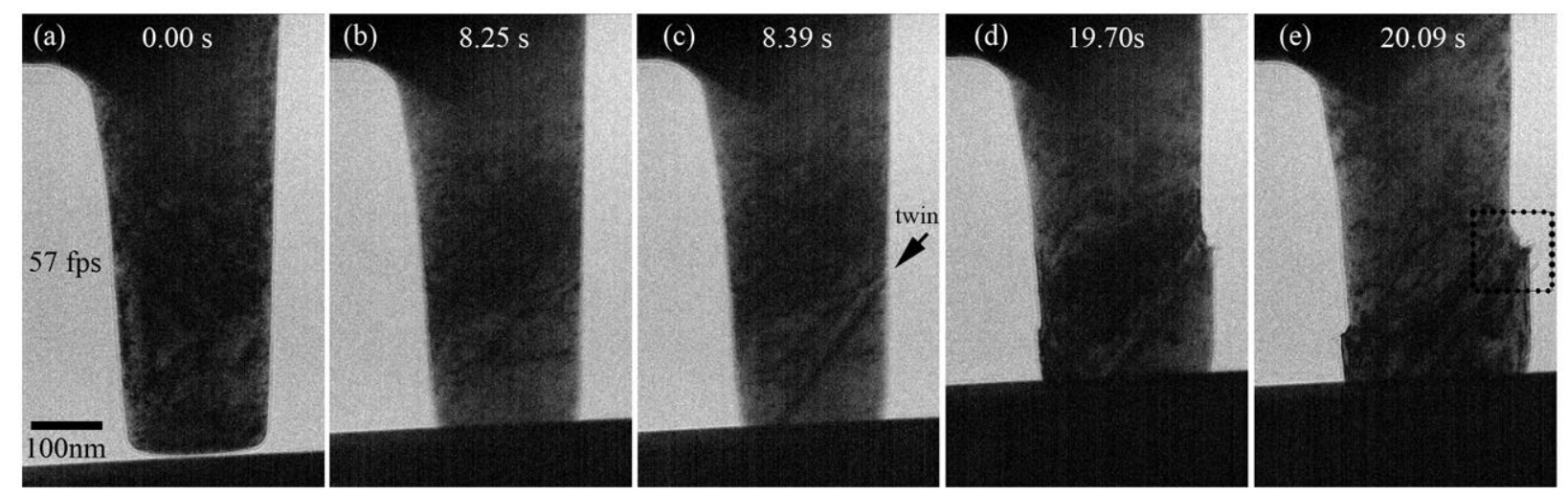

Figure 1. Series of TEM bright field images of twining process in $\mathrm{Cu}$ nanopillar from video stream recorded at a frame rate of 57 frames per second. Note that the time scale is not linear.

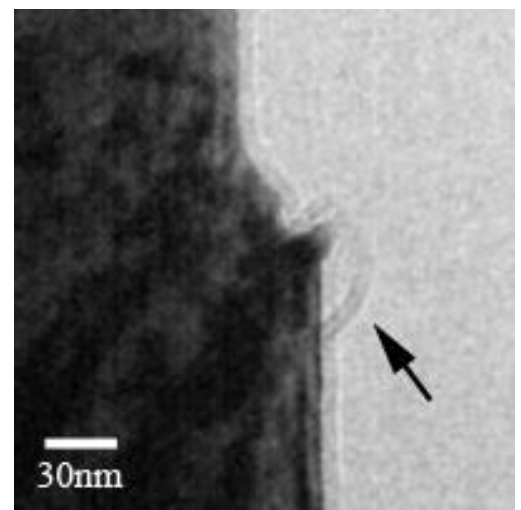

Figure 2. TEM bright field image of damaged layer peeled off from the surface, where twining happened as framed in Fig. 1(e).

Instrumentation: DE-12 DDD camera system, Hitachi H-9500 E-TEM, Hysitron PI 95 in-situ TEM holder. 